\title{
On a half-discrete Hilbert-type inequality similar to Mulholland's inequality
}

\section{Zhenxiao Huang ${ }^{1}$ and Bicheng Yang ${ }^{2 *}$}

\section{"Correspondence:}

bcyang@gdei.edu.cn; bcyang818@163.com

2Department of Mathematics,

Guangdong University of

Education, Guangzhou, Guangdong

510303, P.R. China

Full list of author information is

available at the end of the article

\begin{abstract}
By using the way of weight functions and Hadamard's inequality, a half-discrete Hilbert-type inequality similar to Mulholland's inequality with a best constant factor is given. The extension with multi-parameters, the equivalent forms as well as the operator expressions are also considered.
\end{abstract}

MSC: $26 \mathrm{D} 15$

Keywords: Hilbert-type inequality; weight function; equivalent form

\section{Introduction}

Assuming that $f, g \in L^{2}\left(R_{+}\right),\|f\|=\left\{\int_{0}^{\infty} f^{2}(x) d x\right\}^{\frac{1}{2}}>0,\|g\|>0$, we have the following Hilbert integral inequality (cf. [1]):

$$
\int_{0}^{\infty} \int_{0}^{\infty} \frac{f(x) g(y)}{x+y} d x d y<\pi\|f\|\|g\|
$$

where the constant factor $\pi$ is the best possible. If $a=\left\{a_{n}\right\}_{n=1}^{\infty}, b=\left\{b_{n}\right\}_{n=1}^{\infty} \in l^{2},\|a\|=$ $\left\{\sum_{n=1}^{\infty} a_{n}^{2}\right\}^{\frac{1}{2}}>0,\|b\|>0$, then we still have the following discrete Hilbert inequality:

$$
\sum_{m=1}^{\infty} \sum_{n=1}^{\infty} \frac{a_{m} b_{n}}{m+n}<\pi\|a\|\|b\|
$$

with the same best constant factor $\pi$. Inequalities (1) and (2) are important in analysis and its applications ( $c f$. [2-4]). Also we have the following Mulholland inequality with the same best constant factor $(c f .[1,5])$ :

$$
\sum_{m=2}^{\infty} \sum_{n=2}^{\infty} \frac{a_{m} b_{n}}{\ln m n}<\pi\left\{\sum_{m=2}^{\infty} m a_{m}^{2} \sum_{n=2}^{\infty} n b_{n}^{2}\right\}^{\frac{1}{2}} .
$$

In 1998, by introducing an independent parameter $\lambda \in(0,1]$, Yang [6] gave an extension of (1). By generalizing the results from [6], Yang [7] gave some best extensions of (1) and (2) as follows: If $p>1, \frac{1}{p}+\frac{1}{q}=1, \lambda_{1}+\lambda_{2}=\lambda, k_{\lambda}(x, y)$ is a non-negative homogeneous function of degree $-\lambda$ with $k\left(\lambda_{1}\right)=\int_{0}^{\infty} k_{\lambda}(t, 1) t^{\lambda_{1}-1} d t \in R_{+}, \phi(x)=x^{p\left(1-\lambda_{1}\right)-1}, \psi(x)=$ $x^{q\left(1-\lambda_{2}\right)-1}, f(\geq 0) \in L_{p, \phi}\left(R_{+}\right)=\left\{f \mid\|f\|_{p, \phi}:=\left\{\int_{0}^{\infty} \phi(x)|f(x)|^{p} d x\right\}^{\frac{1}{p}}<\infty\right\}, g(\geq 0) \in L_{q, \psi}\left(R_{+}\right)$, 
$\|f\|_{p, \phi},\|g\|_{q, \psi}>0$, then

$$
\int_{0}^{\infty} \int_{0}^{\infty} k_{\lambda}(x, y) f(x) g(y) d x d y<k\left(\lambda_{1}\right)\|f\|_{p, \phi}\|g\|_{q, \psi},
$$

where the constant factor $k\left(\lambda_{1}\right)$ is the best possible. Moreover, if $k_{\lambda}(x, y)$ is finite and $k_{\lambda}(x, y) x^{\lambda_{1}-1}\left(k_{\lambda}(x, y) y^{\lambda_{2}-1}\right)$ is decreasing for $x>0(y>0)$, then for $a_{m}, b_{n} \geq 0, a=\left\{a_{m}\right\}_{m=1}^{\infty} \in$ $l_{p, \phi}=\left\{a \mid\|a\|_{p, \phi}:=\left\{\sum_{n=1}^{\infty} \phi(n)\left|a_{n}\right|^{p}\right\}^{\frac{1}{p}}<\infty\right\}, b=\left\{b_{n}\right\}_{n=1}^{\infty} \in l_{q, \psi},\|a\|_{p, \phi},\|b\|_{q, \psi}>0$, we have

$$
\sum_{m=1}^{\infty} \sum_{n=1}^{\infty} k_{\lambda}(m, n) a_{m} b_{n}<k\left(\lambda_{1}\right)\|a\|_{p, \phi}\|b\|_{q, \psi}
$$

with the same best constant factor $k\left(\lambda_{1}\right)$. Clearly, for $p=q=2, \lambda=1, k_{1}(x, y)=\frac{1}{x+y}, \lambda_{1}=$ $\lambda_{2}=\frac{1}{2}$, (4) reduces to (1), while (5) reduces to (2). Some other results about Hilbert-type inequalities are provided by $[5,8-16]$.

On the topic of half-discrete Hilbert-type inequalities with the general nonhomogeneous kernels, Hardy et al. provided a few results in Theorem 351 of [1]. But they did not prove that the constant factors in the inequalities are the best possible. Moreover, Yang [17] gave an inequality with the particular kernel $\frac{1}{(1+n x)^{\lambda}}$ and an interval variable, and proved that the constant factor is the best possible. Recently, [18] and [19] gave the following half-discrete Hilbert inequality with the best constant factor $\pi$ :

$$
\int_{0}^{\infty} f(x) \sum_{n=1}^{\infty} \frac{a_{n}}{(x+n)^{\lambda}} d x<\pi\|f\|\|a\|
$$

In this paper, by using the way of weight functions and Hadamard's inequality, a halfdiscrete Hilbert-type inequality similar to (3) and (6) with the best constant factor is given as follows:

$$
\int_{0}^{\infty} f(x) \sum_{n=1}^{\infty} \frac{a_{n}}{\ln e\left(n+\frac{1}{2}\right)^{x}} d x<\pi\|f\|\left\{\sum_{n=1}^{\infty}\left(n+\frac{1}{2}\right) a_{n}^{2}\right\}^{\frac{1}{2}} .
$$

Moreover, the best extension of (7) with multi-parameters, some equivalent forms as well as the operator expressions are considered.

\section{Some lemmas}

Lemma 1 If $0<\lambda \leq 2, \alpha \geq \frac{1}{2}$, setting weight functions $\omega(n)$ and $\varpi(x)$ as follows:

$$
\begin{aligned}
& \omega(n):=\ln ^{\frac{\lambda}{2}}(n+\alpha) \int_{0}^{\infty} \frac{x^{\frac{\lambda}{2}-1}}{\ln ^{\lambda} e(n+\alpha)^{x}} d x, \quad n \in \mathbf{N}, \\
& \varpi(x):=x^{\frac{\lambda}{2}} \sum_{n=1}^{\infty} \frac{\ln ^{\frac{\lambda}{2}-1}(n+\alpha)}{(n+\alpha) \ln ^{\lambda} e(n+\alpha)^{x}}, \quad x \in(0, \infty),
\end{aligned}
$$

we have

$$
\varpi(x)<\omega(n)=B\left(\frac{\lambda}{2}, \frac{\lambda}{2}\right) .
$$


Proof Substitution of $t=x \ln (n+\alpha)$ in (8), by calculation, yields

$$
\omega(n)=\int_{0}^{\infty} \frac{1}{(1+t)^{\lambda}} t^{\frac{\lambda}{2}-1} d t=B\left(\frac{\lambda}{2}, \frac{\lambda}{2}\right) .
$$

Since, for fixed $x>0$ and in view of the conditions,

$$
\begin{aligned}
h(x, y) & :=\frac{\ln ^{\frac{\lambda}{2}-1}(y+\alpha)}{(y+\alpha) \ln ^{\lambda} e(y+\alpha)^{x}} \\
& =\frac{\ln ^{\frac{\lambda}{2}}-1(y+\alpha)}{(y+\alpha)[1+x \ln (y+\alpha)]^{\lambda}}
\end{aligned}
$$

is decreasing and strictly convex for $y \in\left(\frac{1}{2}, \infty\right)$, then by Hadamard's inequality (cf. [20]), we find

$$
\begin{gathered}
\varpi(x)<x^{\frac{\lambda}{2}} \int_{\frac{1}{2}}^{\infty} \frac{\ln ^{\frac{\lambda}{2}-1}(y+\alpha)}{(y+\alpha)[1+x \ln (y+\alpha)]^{\lambda}} d y \\
\stackrel{t=x \ln (y+\alpha)}{=} \int_{x \ln \left(\frac{1}{2}+\alpha\right)}^{\infty} \frac{t^{\frac{\lambda}{2}-1}}{(1+t)^{\lambda}} d t \leq B\left(\frac{\lambda}{2}, \frac{\lambda}{2}\right),
\end{gathered}
$$

namely, (10) follows.

Lemma 2 Let the assumptions of Lemma 1 be fulfilled and, additionally, let $p>1, \frac{1}{p}+\frac{1}{q}=1$, $a_{n} \geq 0, n \in \mathbf{N}, f(x)$ be a non-negative measurable function in $(0, \infty)$. Then we have the following inequalities:

$$
\begin{aligned}
J & :=\left\{\sum_{n=1}^{\infty} \frac{\ln \frac{p \lambda}{2}-1}{n+\alpha}\left[\int_{0}^{\infty} \frac{f(x)}{\ln ^{\lambda} e(n+\alpha)^{x}} d x\right]^{p}\right\}^{\frac{1}{p}} \\
& \leq\left[B\left(\frac{\lambda}{2}, \frac{\lambda}{2}\right)\right]^{\frac{1}{q}}\left\{\int_{0}^{\infty} \varpi(x) x^{p\left(1-\frac{\lambda}{2}\right)-1} f^{p}(x) d x\right\}^{\frac{1}{p}}, \\
L_{1} & :=\left\{\int_{0}^{\infty} \frac{x^{\frac{q \lambda}{2}}-1}{[\varpi(x)]^{q-1}}\left[\sum_{n=1}^{\infty} \frac{a_{n}}{\ln ^{\lambda} e(n+\alpha)^{x}}\right]^{q} d x\right\}^{\frac{1}{q}} \\
& \leq\left\{B\left(\frac{\lambda}{2}, \frac{\lambda}{2}\right) \sum_{n=1}^{\infty}(n+\alpha)^{q-1} \ln ^{q\left(1-\frac{\lambda}{2}\right)-1}(n+\alpha) a_{n}^{q}\right\}^{\frac{1}{q}} .
\end{aligned}
$$

Proof By Hölder's inequality (cf. [20]) and (10), it follows

$$
\begin{aligned}
& {\left[\int_{0}^{\infty} \frac{f(x) d x}{\ln ^{\lambda} e(n+\alpha)^{x}}\right]^{p}} \\
& \quad=\left\{\int_{0}^{\infty} \frac{1}{\ln ^{\lambda} e(n+\alpha)^{x}}\left[\frac{x^{\left(1-\frac{\lambda}{2}\right) / q}}{\ln ^{\left(1-\frac{\lambda}{2}\right) / p}(n+\alpha)} \frac{f(x)}{(n+\alpha)^{\frac{1}{p}}}\right]\left[\frac{\ln ^{\left(1-\frac{\lambda}{2}\right) / p}(n+\alpha)}{x^{\left(1-\frac{\lambda}{2}\right) / q}}(n+\alpha)^{\frac{1}{p}}\right] d x\right\}^{p} \\
& \quad \leq \int_{0}^{\infty} \frac{\ln ^{\frac{\lambda}{2}-1}(n+\alpha)}{\ln ^{\lambda} e(n+\alpha)^{x}} \frac{x^{\left(1-\frac{\lambda}{2}\right)(p-1)} f^{p}(x) d x}{n+\alpha}\left\{\int_{0}^{\infty} \frac{(n+\alpha)^{q-1}}{\ln ^{\lambda} e(n+\alpha)^{x}} \frac{\ln ^{\left(1-\frac{\lambda}{2}\right)(q-1)}(n+\alpha)}{x^{1-\frac{\lambda}{2}}} d x\right\}^{p-1}
\end{aligned}
$$




$$
\begin{aligned}
& =\left\{\frac{\omega(n)(n+\alpha)^{q-1}}{\ln ^{q\left(\frac{\lambda}{2}-1\right)+1}(n+\alpha)}\right\}^{p-1} \int_{0}^{\infty} \frac{\ln ^{\frac{\lambda}{2}-1}(n+\alpha)}{\ln ^{\lambda} e(n+\alpha)^{x}} \frac{x^{\left(1-\frac{\lambda}{2}\right)(p-1)} f^{p}(x) d x}{n+\alpha} \\
& =\left[B\left(\frac{\lambda}{2}, \frac{\lambda}{2}\right)\right]^{p-1} \frac{n+\alpha}{\ln ^{\frac{p \lambda}{2}-1}(n+\alpha)} \int_{0}^{\infty} \frac{\ln ^{\frac{\lambda}{2}-1}(n+\alpha)}{\ln ^{\lambda} e(n+\alpha)^{x}} \frac{x^{\left(1-\frac{\lambda}{2}\right)(p-1)} f^{p}(x) d x}{n+\alpha} .
\end{aligned}
$$

Then by the Lebesgue term-by-term integration theorem ( $c f$. [21]), we have

$$
\begin{aligned}
J & \leq\left[B\left(\frac{\lambda}{2}, \frac{\lambda}{2}\right)\right]^{\frac{1}{q}}\left\{\sum_{n=1}^{\infty} \int_{0}^{\infty} \frac{\ln ^{\frac{\lambda}{2}-1}(n+\alpha)}{\ln ^{\lambda} e(n+\alpha)^{x}} \frac{x^{\left(1-\frac{\lambda}{2}\right)(p-1)} f^{p}(x) d x}{n+\alpha}\right\}^{\frac{1}{p}} \\
& =\left[B\left(\frac{\lambda}{2}, \frac{\lambda}{2}\right)\right]^{\frac{1}{q}}\left\{\int_{0}^{\infty} \sum_{n=1}^{\infty} \frac{\ln ^{\frac{\lambda}{2}-1}(n+\alpha)}{\ln ^{\lambda} e((n+\alpha))^{x}} \frac{x^{\left(1-\frac{\lambda}{2}\right)(p-1)} f^{p}(x) d x}{n+\alpha}\right\}^{\frac{1}{p}} \\
& =\left[B\left(\frac{\lambda}{2}, \frac{\lambda}{2}\right)\right]^{\frac{1}{q}}\left\{\int_{0}^{\infty} \varpi(x) x^{p\left(1-\frac{\lambda}{2}\right)-1} f^{p}(x) d x\right\}^{\frac{1}{p}},
\end{aligned}
$$

and (11) follows. Still by Hölder's inequality, we have

$$
\begin{aligned}
& {\left[\sum_{n=1}^{\infty} \frac{a_{n}}{\ln ^{\lambda} e(n+\alpha)^{x}}\right]^{q}} \\
& \quad=\left\{\sum_{n=1}^{\infty} \frac{1}{\ln ^{\lambda} e(n+\alpha)^{x}}\left[\frac{x^{\left(1-\frac{\lambda}{2}\right) / q}}{\ln ^{\left(1-\frac{\lambda}{2}\right) / p}(n+\alpha)} \frac{1}{(n+\alpha)^{\frac{1}{p}}}\right]\left[\frac{\ln ^{\left(1-\frac{\lambda}{2}\right) / p}(n+\alpha)}{x^{\left(1-\frac{\lambda}{2}\right) / q}}(n+\alpha)^{\frac{1}{p}} a_{n}\right]\right\}^{q} \\
& \quad \leq\left\{\sum_{n=1}^{\infty} \frac{\ln ^{\frac{\lambda}{2}-1}(n+\alpha)}{\ln ^{\lambda} e(n+\alpha)^{x}} \frac{x^{\left(1-\frac{\lambda}{2}\right)(p-1)}}{(n+\alpha)}\right\}^{q-1} \sum_{n=1}^{\infty} \frac{(n+\alpha)^{q-1}}{\ln ^{\lambda} e(n+\alpha)^{x}} \frac{\ln ^{\left(1-\frac{\lambda}{2}\right)(q-1)}(n+\alpha)}{x^{1-\frac{\lambda}{2}}} a_{n}^{q} \\
& \quad=\frac{[\varpi(x)]^{q-1}}{x^{\frac{q \lambda}{2}-1}} \sum_{n=1}^{\infty} \frac{(n+\alpha)^{q-1}}{\ln ^{\lambda} e(n+\alpha)^{x}} x^{\frac{\lambda}{2}-1} \ln ^{\left(1-\frac{\lambda}{2}\right)(q-1)}(n+\alpha) a_{n}^{q} .
\end{aligned}
$$

Then by the Lebesgue term-by-term integration theorem, we have

$$
\begin{aligned}
L_{1} & \leq\left\{\int_{0}^{\infty} \sum_{n=1}^{\infty} \frac{(n+\alpha)^{q-1}}{\ln ^{\lambda} e(n+\alpha)^{x}} x^{\frac{\lambda}{2}-1} \ln ^{\left(1-\frac{\lambda}{2}\right)(q-1)}(n+\alpha) a_{n}^{q} d x\right\}^{\frac{1}{q}} \\
& =\left\{\sum_{n=1}^{\infty}\left[\ln ^{\frac{\lambda}{2}}(n+\alpha) \int_{0}^{\infty} \frac{x^{\frac{\lambda}{2}-1} d x}{\ln ^{\lambda} e(n+\alpha)^{x}}\right](n+\alpha)^{q-1} \ln ^{q\left(1-\frac{\lambda}{2}\right)-1}(n+\alpha) a_{n}^{q}\right\}^{\frac{1}{q}} \\
& =\left\{\sum_{n=1}^{\infty} \omega(n)(n+\alpha)^{q-1} \ln ^{q\left(1-\frac{\lambda}{2}\right)-1}(n+\alpha) a_{n}^{q}\right\}^{\frac{1}{q}},
\end{aligned}
$$

and then in view of (10), inequality (12) follows.

\section{Main results}

We introduce two functions

$$
\Phi(x):=x^{p\left(1-\frac{\lambda}{2}\right)-1} \quad(x>0) \quad \text { and } \quad \Psi(n):=(n+\alpha)^{q-1} \ln ^{q\left(1-\frac{\lambda}{2}\right)-1}(n+\alpha) \quad(n \in \mathbf{N}) \text {, }
$$

wherefrom, $[\Phi(x)]^{1-q}=x^{\frac{q \lambda}{2}-1}$, and $[\Psi(n)]^{1-p}=\frac{\ln \frac{p \lambda}{2}-1}{n+\alpha+\alpha)}$. 
Theorem 1 If $0<\lambda \leq 2, \alpha \geq \frac{1}{2}, p>1, \frac{1}{p}+\frac{1}{q}=1, f(x), a_{n} \geq 0, f \in L_{p, \Phi}\left(R_{+}\right), a=\left\{a_{n}\right\}_{n=1}^{\infty} \in l_{q, \Psi}$, $\|f\|_{p, \Phi}>0$ and $\|a\|_{q, \Psi}>0$, then we have the following equivalent inequalities:

$$
\begin{aligned}
I & :=\sum_{n=1}^{\infty} \int_{0}^{\infty} \frac{a_{n} f(x) d x}{\ln ^{\lambda} e(n+\alpha)^{x}} \\
& =\int_{0}^{\infty} \sum_{n=1}^{\infty} \frac{a_{n} f(x) d x}{\ln ^{\lambda} e(n+\alpha)^{x}}<B\left(\frac{\lambda}{2}, \frac{\lambda}{2}\right)\|f\|_{p, \Phi}\|a\|_{q, \Psi}, \\
J & =\left\{\sum_{n=1}^{\infty}[\Psi(n)]^{1-p}\left[\int_{0}^{\infty} \frac{f(x)}{\ln ^{\lambda} e(n+\alpha)^{x}} d x\right]^{p}\right\}^{\frac{1}{p}} \\
& <B\left(\frac{\lambda}{2}, \frac{\lambda}{2}\right)\|f\|_{p, \Phi}, \\
L & :=\left\{\int_{0}^{\infty}[\Phi(x)]^{1-q}\left[\sum_{n=1}^{\infty} \frac{a_{n}}{\ln ^{\lambda} e(n+\alpha)^{x}}\right]^{q} d x\right\}^{\frac{1}{q}} \\
& <B\left(\frac{\lambda}{2}, \frac{\lambda}{2}\right)\|a\|_{q, \Psi},
\end{aligned}
$$

where the constant $B\left(\frac{\lambda}{2}, \frac{\lambda}{2}\right)$ is the best possible in the above inequalities.

Proof By the Lebesgue term-by-term integration theorem, there are two expressions for $I$ in (13). In view of (11), for $\varpi(x)<B\left(\frac{\lambda}{2}, \frac{\lambda}{2}\right)$, we have (14). By Hölder's inequality, we have

$$
I=\sum_{n=1}^{\infty}\left[\Psi^{\frac{-1}{q}}(n) \int_{0}^{\infty} \frac{1}{\ln ^{\lambda} e(n+\alpha)^{x}} f(x) d x\right]\left[\Psi^{\frac{1}{q}}(n) a_{n}\right] \leq J\|a\|_{q, \Psi} .
$$

Then by (14), we have (13). On the other hand, assuming that (13) is valid, setting

$$
a_{n}:=[\Psi(n)]^{1-p}\left[\int_{0}^{\infty} \frac{1}{\ln ^{\lambda} e(n+\alpha)^{x}} f(x) d x\right]^{p-1}, \quad n \in \mathbf{N},
$$

then $J^{p-1}=\|a\|_{q, \Psi}$. By (11), we find $J<\infty$. If $J=0$, then (14) is trivially valid; if $J>0$, then by (13), we have

$$
\begin{aligned}
& \|a\|_{q, \Psi}^{q}=J^{p}=I<B\left(\frac{\lambda}{2}, \frac{\lambda}{2}\right)\|f\|_{p, \Phi}\|a\|_{q, \Psi}, \quad \text { i.e. } \\
& \|a\|_{q, \Psi}^{q-1}=J<B\left(\frac{\lambda}{2}, \frac{\lambda}{2}\right)\|f\|_{p, \Phi}
\end{aligned}
$$

that is, (14) is equivalent to (13). In view of (12), for $[\varpi(x)]^{1-q}>\left[B\left(\frac{\lambda}{2}, \frac{\lambda}{2}\right)\right]^{1-q}$, we have (15). By Hölder's inequality, we find

$$
I=\int_{0}^{\infty}\left[\Phi^{\frac{1}{p}}(x) f(x)\right]\left[\Phi^{\frac{-1}{p}}(x) \sum_{n=1}^{\infty} \frac{1}{\ln ^{\lambda} e(n+\alpha)^{x}} a_{n}\right] d x \leq\|f\|_{p, \Phi} L .
$$

Then by (15), we have (13). On the other hand, assuming that (13) is valid, setting

$$
f(x):=[\Phi(x)]^{1-q}\left[\sum_{n=1}^{\infty} \frac{1}{\ln ^{\lambda} e(n+\alpha)^{x}} a_{n}\right]^{q-1}, \quad x \in(0, \infty),
$$


then $L^{q-1}=\|f\|_{p, \Phi}$. By (12), we find $L<\infty$. If $L=0$, then (15) is trivially valid; if $L>0$, then by (13), we have

$$
\begin{aligned}
& \|f\|_{p, \Phi}^{p}=L^{q}=I<B\left(\frac{\lambda}{2}, \frac{\lambda}{2}\right)\|f\|_{p, \Phi}\|a\|_{q, \Psi}, \quad \text { i.e. } \\
& \|f\|_{p, \Phi}^{p-1}=L<B\left(\frac{\lambda}{2}, \frac{\lambda}{2}\right)\|a\|_{q, \Psi}
\end{aligned}
$$

that is, (15) is equivalent to (13). Hence, inequalities (13), (14) and (15) are equivalent.

For $0<\varepsilon<\frac{p \lambda}{2}$, setting $\widetilde{f}(x)=x^{\frac{\lambda}{2}+\frac{\varepsilon}{p}-1}, x \in(0,1) ; \widetilde{f}(x)=0, x \in[1, \infty)$, and $\widetilde{a}_{n}=\frac{1}{n+\alpha} \times$ $\ln ^{\frac{\lambda}{2}-\frac{\varepsilon}{q}-1}(n+\alpha), n \in \mathbf{N}$, if there exists a positive number $k\left(\leq B\left(\frac{\lambda}{2}, \frac{\lambda}{2}\right)\right)$ such that (13) is valid as we replace $B\left(\frac{\lambda}{2}, \frac{\lambda}{2}\right)$ with $k$, then, in particular, it follows

$$
\begin{aligned}
& \widetilde{I}:=\sum_{n=1}^{\infty} \int_{0}^{\infty} \frac{1}{\ln ^{\lambda} e(n+\alpha)^{x}} \widetilde{a}_{n} \widetilde{f}(x) d x<k\|\tilde{f}\|_{p, \Phi}\|\widetilde{a}\|_{q, \Psi} \\
& =k\left\{\int_{0}^{1} \frac{d x}{x^{-\varepsilon+1}}\right\}^{\frac{1}{p}}\left\{\frac{1}{(1+\alpha) \ln ^{\varepsilon+1}(1+\alpha)}+\sum_{n=2}^{\infty} \frac{1}{(n+\alpha) \ln ^{\varepsilon+1}(n+\alpha)}\right\}^{\frac{1}{q}} \\
& <k\left(\frac{1}{\varepsilon}\right)^{\frac{1}{p}}\left\{\frac{1}{(1+\alpha) \ln ^{\varepsilon+1}(1+\alpha)}+\int_{1}^{\infty} \frac{1}{(x+\alpha) \ln ^{\varepsilon+1}(x+\alpha)} d x\right\}^{\frac{1}{q}} \\
& =\frac{k}{\varepsilon}\left\{\frac{\varepsilon}{(1+\alpha) \ln ^{\varepsilon+1}(1+\alpha)}+\frac{1}{\ln ^{\varepsilon}(1+\alpha)}\right\}^{\frac{1}{q}} \text {, } \\
& \widetilde{I}=\sum_{n=1}^{\infty} \frac{1}{n+\alpha} \ln ^{\frac{\lambda}{2}-\frac{\varepsilon}{q}-1}(n+\alpha) \int_{0}^{1} \frac{1}{\ln ^{\lambda} e(n+\alpha)^{x}} x^{\frac{\lambda}{2}+\frac{\varepsilon}{p}-1} d x \\
& \stackrel{t=x \ln (n+\alpha)}{=} \sum_{n=1}^{\infty} \frac{1}{(n+\alpha) \ln ^{\varepsilon+1}(n+\alpha)} \int_{0}^{\ln (n+\alpha)} \frac{1}{(t+1)^{\lambda}} t^{\frac{\lambda}{2}+\frac{\varepsilon}{p}-1} d t \\
& =B\left(\frac{\lambda}{2}+\frac{\varepsilon}{p}, \frac{\lambda}{2}-\frac{\varepsilon}{p}\right) \sum_{n=1}^{\infty} \frac{1}{(n+\alpha) \ln ^{\varepsilon+1}(n+\alpha)}-A(\varepsilon) \\
& >\quad B\left(\frac{\lambda}{2}+\frac{\varepsilon}{p}, \frac{\lambda}{2}-\frac{\varepsilon}{p}\right) \int_{1}^{\infty} \frac{1}{(y+\alpha) \ln ^{\varepsilon+1}(y+\alpha)} d y-A(\varepsilon) \\
& =\frac{1}{\varepsilon \ln ^{\varepsilon}(1+\alpha)} B\left(\frac{\lambda}{2}+\frac{\varepsilon}{p}, \frac{\lambda}{2}-\frac{\varepsilon}{p}\right)-A(\varepsilon), \\
& A(\varepsilon):=\sum_{n=1}^{\infty} \frac{1}{(n+\alpha) \ln ^{\varepsilon+1}(n+\alpha)} \int_{\ln (n+\alpha)}^{\infty} \frac{1}{(t+1)^{\lambda}} t^{\frac{\lambda}{2}+\frac{\varepsilon}{p}-1} d t .
\end{aligned}
$$

We find

$$
\begin{aligned}
0 & <A(\varepsilon) \leq \sum_{n=1}^{\infty} \frac{1}{(n+\alpha) \ln ^{\varepsilon+1}(n+\alpha)} \int_{\ln (n+\alpha)}^{\infty} \frac{1}{t^{\lambda}} t^{\frac{\lambda}{2}+\frac{\varepsilon}{p}-1} d t \\
& =\frac{1}{\frac{\lambda}{2}-\frac{\varepsilon}{p}} \sum_{n=1}^{\infty} \frac{1}{(n+\alpha) \ln ^{\frac{\lambda}{2}+\frac{\varepsilon}{q}+1}(n+\alpha)}<\infty,
\end{aligned}
$$


and then $A(\varepsilon)=O(1)\left(\varepsilon \rightarrow 0^{+}\right)$. Hence by (18) and (19), it follows

$$
\begin{aligned}
& \frac{1}{\ln ^{\varepsilon}(1+\alpha)} B\left(\frac{\lambda}{2}+\frac{\varepsilon}{p}, \frac{\lambda}{2}-\frac{\varepsilon}{p}\right)-\varepsilon O(1) \\
& \quad<k\left\{\frac{\varepsilon}{(1+\alpha) \ln ^{\varepsilon+1}(1+\alpha)}+\frac{1}{\ln ^{\varepsilon}(1+\alpha)}\right\}^{\frac{1}{q}},
\end{aligned}
$$

and $B\left(\frac{\lambda}{2}, \frac{\lambda}{2}\right) \leq k\left(\varepsilon \rightarrow 0^{+}\right)$. Hence $k=B\left(\frac{\lambda}{2}, \frac{\lambda}{2}\right)$ is the best value of (13).

By equivalence, the constant factor $B\left(\frac{\lambda}{2}, \frac{\lambda}{2}\right)$ in (14) and (15) is the best possible. Otherwise, we can imply a contradiction by (16) and (17) that the constant factor in (13) is not the best possible.

Remark 1 (i) Define the first type half-discrete Hilbert-type operator $T_{1}: L_{p, \Phi}\left(R_{+}\right) \rightarrow$ $l_{p, \Psi^{1-p}}$ as follows: For $f \in L_{p, \Phi}\left(R_{+}\right)$, we define $T_{1} f \in l_{p, \Psi^{1-p}}$, satisfying

$$
T_{1} f(n)=\int_{0}^{\infty} \frac{1}{\ln ^{\lambda} e(n+\alpha)^{x}} f(x) d x, \quad n \in \mathbf{N} .
$$

Then by (14) it follows $\left\|T_{1} f\right\|_{p . \Psi^{1-p}} \leq B\left(\frac{\lambda}{2}, \frac{\lambda}{2}\right)\|f\|_{p, \Phi}$, and then $T_{1}$ is a bounded operator with $\left\|T_{1}\right\| \leq B\left(\frac{\lambda}{2}, \frac{\lambda}{2}\right)$. Since by Theorem 1 the constant factor in (14) is the best possible, we have $\left\|T_{1}\right\|=B\left(\frac{\lambda}{2}, \frac{\lambda}{2}\right)$.

(ii) Define the second type half-discrete Hilbert-type operator $T_{2}: l_{q, \Psi} \rightarrow L_{q, \Phi^{1-q}}\left(R_{+}\right)$as follows: For $a \in l_{q, \Psi}$, we define $T_{2} a \in L_{q, \Phi^{1-q}}\left(R_{+}\right)$, satisfying

$$
T_{2} a(x)=\sum_{n=1}^{\infty} \frac{1}{\ln ^{\lambda} e(n+\alpha)^{x}} a_{n}, \quad x \in(0, \infty) .
$$

Then by (15) it follows $\left\|T_{2} a\right\|_{q, \Phi^{1-q}} \leq B\left(\frac{\lambda}{2}, \frac{\lambda}{2}\right)\|a\|_{q, \Psi}$, and then $T_{2}$ is a bounded operator with $\left\|T_{2}\right\| \leq B\left(\frac{\lambda}{2}, \frac{\lambda}{2}\right)$. Since by Theorem 1 the constant factor in (15) is the best possible, we have $\left\|T_{2}\right\|=B\left(\frac{\lambda}{2}, \frac{\lambda}{2}\right)$.

Remark 2 For $p=q=2, \lambda=1, \lambda_{1}=\lambda_{2}=\frac{1}{2}, \alpha=\frac{1}{2}$ in (13), (14) and (15), we have (7) and the following equivalent inequalities:

$$
\begin{aligned}
& \left\{\sum_{n=1}^{\infty} \frac{1}{n+\frac{1}{2}}\left[\int_{0}^{\infty} \frac{f(x)}{\ln e\left(n+\frac{1}{2}\right)^{x}} d x\right]^{2}\right\}^{\frac{1}{2}}<\pi\|f\|, \\
& \left\{\int_{0}^{\infty}\left[\sum_{n=1}^{\infty} \frac{a_{n}}{\ln e\left(n+\frac{1}{2}\right)^{x}}\right]^{2} d x\right\}^{\frac{1}{2}}<\pi\left\{\sum_{n=1}^{\infty}\left(n+\frac{1}{2}\right) a_{n}^{2}\right\}^{\frac{1}{2}} .
\end{aligned}
$$




\section{Author details}

'Basic Education College of Zhanjiang Normal University, Zhanjiang, Guangdong 524037, P.R. China. ${ }^{2}$ Department of Mathematics, Guangdong University of Education, Guangzhou, Guangdong 510303, P.R. China.

\section{Acknowledgements}

This work is supported by 2012 Knowledge Construction Special Foundation Item of Guangdong Institution of Higher Learning College and University (No. 2012KJCX0079).

\section{Received: 23 January 2013 Accepted: 30 April 2013 Published: 7 June 2013}

\section{References}

1. Hardy, GH, Littlewood, JE, Pólya, G: Inequalities. Cambridge University Press, Cambridge (1934)

2. Mitrinović, DS, Pečarić, JE, Fink, AM: Inequalities Involving Functions and Their Integrals and Derivatives. Kluwer Acaremic, Boston (1991)

3. Yang, B: Hilbert-Type Integral Inequalities. Bentham Science Publishers, Sharjah (2009)

4. Yang, B: Discrete Hilbert-Type Inequalities. Bentham Science Publishers, Sharjah (2011)

5. Yang, B: On a new extension of Hilbert's inequality with some parameters. Acta Math. Hung. 108(4), 337-350 (2005)

6. Yang, B: On Hilbert's integral inequality. J. Math. Anal. Appl. 220, 778-785 (1998)

7. Yang, B: The Norm of Operator and Hilbert-Type Inequalities. Science Press, Beijin (2009).

8. Yang, B, Brnetić, I, Krnić, M, Pečarić, J: Generalization of Hilbert and Hardy-Hilbert integral inequalities. Math. Inequal. Appl. 8(2), 259-272 (2005)

9. Krnić, M, Pečarić, J: Hilbert's inequalities and their reverses. Publ. Math. (Debr.) 67(3-4), 315-331 (2005)

10. Jin, J, Debnath, L: On a Hilbert-type linear series operator and its applications. J. Math. Anal. Appl. 371, 691-704 (2010)

11. Azar, L: On some extensions of Hardy-Hilbert's inequality and applications. J. Inequal. Appl. 2009, Article ID 546829 (2009)

12. Yang, B, Rassias, TM: On the way of weight coefficient and research for Hilbert-type inequalities. Math. Inequal. Appl. 6(4), 625-658 (2003)

13. Arpad, B, Choonghong, O: Best constant for certain multilinear integral operator. J. Inequal. Appl. 2006, Article ID $28582(2006)$

14. Kuang, J, Debnath, L: On Hilbert's type inequalities on the weighted Orlicz spaces. Pac. J. Appl. Math. 1(1), 95-103 (2007)

15. Zhong, W: The Hilbert-type integral inequality with a homogeneous kernel of - $\lambda$-degree. J. Inequal. Appl. 2008 Article ID 917392 (2008)

16. Li, Y, He, B: On inequalities of Hilbert's type. Bull. Aust. Math. Soc. 76(1), 1-13 (2007)

17. Yang, B: A mixed Hilbert-type inequality with a best constant factor. Int. J. Pure Appl. Math. 20(3), $319-328$ (2005)

18. Yang, B: A half-discrete Hilbert's inequality. J. Guangdong Univ. Educ. 31(3), 1-7 (2011)

19. Yang, B, Chen, Q: A half-discrete Hilbert-type inequality with a homogeneous kernel and an extension. J. Inequal. Appl. 2011, 124 (2011). doi:10.1186/1029-242X-2011-124

20. Kuang, J: Applied Inequalities. Shangdong Science Technic Press, Jinan (2004)

21. Kuang, J: Introduction to Real Analysis. Hunan Education Press, Chansha (1996)

doi:10.1186/1029-242X-2013-290

Cite this article as: Huang and Yang: On a half-discrete Hilbert-type inequality similar to Mulholland's inequality.

Journal of Inequalities and Applications 2013 2013:290.

\section{Submit your manuscript to a SpringerOpen ${ }^{\circledR}$ journal and benefit from:}

- Convenient online submission

Rigorous peer review

- Immediate publication on acceptance

- Open access: articles freely available online

- High visibility within the field

- Retaining the copyright to your article 